\title{
Towards Distributed Communications Systems: Relay-Based Wireless Networks
}

\author{
Nikolai Nefedov \\ Nokia Research Center, P.O. Box 407 00045, Finland \\ nikolai.nefedov@nokia.com
}

\begin{abstract}
The increasing demand for high data-rate services stimulates growing deployment of wireless local area network technologies. However, the limited communication range of these technologies makes it difficult and expensive to provide high data-rate services at the periphery of service areas and in environments with harsh channel conditions. Furthermore, this problem even more complicated for future $4 \mathrm{G}$ wireless broadband networks, which are expected to offer data rates up to 100 $\mathrm{Mb} / \mathrm{s}$ for mobile users and up to $1 \mathrm{~Gb} / \mathrm{s}$ for stationary users. To provide a reasonably large coverage for these high data rates the conventional cellular networks appear to be not feasible and it stimulated a search for other architectures.

Recently it is shown that novel concepts such as multi-hop relaying and associated diversity techniques, may significantly enhance the high data rate coverage and increase the throughput beyond-3G networks. In relay-based cellular networks, the mobile terminals which cannot establish (at the required rates) direct links with the base station may communicate to fixed relays or other mobile terminals (e.g., using the unlicensed band) to assist the connection. It allows to substitute a poorquality single-hop wireless link with a composite multi-hop better-quality link whenever possible. In a more general case, a relay-based wireless network may form a distributed communication system where several multi-hop links may be established and different types of re-transmissions (e.g., amplify-forward, decode-forward) are utilized. Besides, due to the broadcasting nature of wireless communications, transmitted signal from a given source may be processed simultaneously for the further retransmission by several relays and/or mobile terminals. These relays, or its subsets, may utilize different forms of cooperation to form, for example, virtual (distributed) antenna arrays. The relay cooperation may be extended beyond beamforming and include also space-time coding concept, where separated relay antennas interconnected with unreliable radio links form a distributed MIMO relaying structure.

Furthermore, relay nodes may combine several incoming source signals and retransmit a combined (coded) version. This recently proposed novel concept, known as network coding, currently attracts the growing attention.

In this talk we overview the recent advances in wireless relay networks, including distributed MIMO relaying, cooperative strategies and network coding.
\end{abstract}

